\title{
Consumo de cafeína e prematuridade
}

\author{
Caffeine intake and prematurity
}

Rita Adriana Gomes de SOUZA ${ }^{1}$

Rosely SICHIERI ${ }^{1}$

\section{RES U M O}

A cafeína (1, 3, 7-trimetilxantina) é uma metilxantina que facilmente atravessa a barreira placentária, com quantidades substanciais passando para o líquido amniótico, sangue do cordão umbilical, plasma e urina dos neonatos. As maiores fontes de cafeína são café, chá, chocolate e refrigerantes do tipo cola. Além disso, cerca de mil drogas prescritas e 2 mil drogas não prescritas contêm cafeína, e 25 dessas drogas podem ser usadas na gravidez. Embora estudos em animais indiquem que a cafeína leve à diminuição no crescimento intrauterino fetal, redução do peso ao nascer, reabsorção fetal e teratogênese, nos estudos epidemiológicos os achados são, ainda, inconclusivos. Pelo fato de os alimentos com cafeína serem amplamente consumidos na gravidez, é importante avaliar se o uso dessa substância está associado com a redução da idade gestacional. Este artigo examina o conhecimento atual do consumo de cafeína durante a gravidez, abordando os estudos epidemiológicos sobre a associação entre consumo de cafeína e prematuridade, as fontes de cafeína e seu consumo na gravidez, a bioquímica, a fisiopatologia e a plausibilidade biológica da associação e as principais limitações dos estudos sobre cafeína e prematuridade.

Termos de indexação: consumo de cafeína, gravidez, prematuridade.

\section{A B S T R A C T}

Caffeine (1, 3, 7-trimethylxanthine) is a methylxanthine that easily crosses the placental barrier, substantial amounts passing into the amniotic fluid, umbilical cord blood, and the plasma and urine of the neonates. The main sources of caffeine are coffee, tea, chocolate and cola soft drinks. Moreover, about 1000 prescribed drugs and 2000 non-prescribed drugs contain caffeine, and 25 of these drugs can be used during pregnancy. Although animal studies indicate that caffeine leads to a decrease in fetal intrauterine growth, low birth weight, fetal re-absorption and teratogenesis, these findings are still inconclusive in the epidemiological studies. Since foods containing caffeine are widely consumed during pregnancy, it is important to evaluate if the consumption of this substance is associated with a reduction in gestational age. This article examines current knowledge on caffeine intake during pregnancy, based on epidemiological studies evaluating the association between caffeine intake and prematurity, the sources of caffeine and its consumption during pregnancy, the biochemistry, physiopathology and biological plausibility of the association, and the main limitations of the studies on caffeine and prematurity.

Indexing terms: caffeine intake, pregnancy, prematurity.

\footnotetext{
1 Instituto de Medicina Social, Universidade do Estado do Rio de Janeiro. Rua São Francisco Xavier, 524, Bloco E, 20550-030, Rio de Janeiro, RJ, Brasil. Correspondência para/Correspondence to: R.A.G. SOUZA. E-mails: <ritadriana@ims.uerj.br> <ritadriana@bol.com.br>.
} 


\section{N T R O D U Ç Ã O}

O interesse pelo estudo da cafeína começou na década de setenta, quando estudos em animais indicaram que a cafeína estaria relacionada à diminuição no crescimento intrauterino fetal, redução do peso ao nascer, reabsorção fetal e teratogênese ${ }^{1,2}$.

Com base nesses achados, ainda na década de setenta, ocorreram os dois primeiros estudos de coorte sobre o consumo de cafeína na gravidez, um nos Estados Unidos ${ }^{3}$ e outro na Alemanha ${ }^{4}$. Ambos mostraram associação entre consumo de cafeína e baixo peso ao nascer (BPN), mas somente o primeiro mostrou associação com a prematuridade.

Desde então, tem-se estudado a relação entre a cafeína e os possíveis efeitos indesejáveis durante a gravidez, sobretudo nos Estados Unidos da América (EUA), onde o Food and Drug Administration (FDA), em 1980, com base nos achados de estudos em animais, sugeriu que as mulheres grávidas evitassem ou diminuíssem o consumo de alimentos e/ou bebidas contendo cafeína ${ }^{5}$. Os resultados de estudos experimentais animais, entretanto, não podem ser aplicados à experiência humana, pois a absorção, metabolismo, excreção e toxicidade à cafeína podem ser diferentes ${ }^{6}$. Além disso, muitos dos efeitos da cafeína em animais ocorreram com o uso de doses que não estão dentro do limite do consumo humano?.

Os Anexos 1 e 2 trazem um resumo dos artigos publicados sobre a associação entre cafeína e prematuridade. As tabelas se referem aos artigos recuperados no Medline sob os temas "preterm", "premature infants", "prematurity", "coffee", "caffeine intake", "consumption".

No Brasil, somente três estudos abordaram o consumo de cafeína na gravidez. O primeiro ${ }^{8}$, realizado em Campinas, mostrou um efeito dose-resposta e restrição de crescimento intra-uterino (RCIU). O segundo, realizado em Pelotas ${ }^{9}$, pesquisou a relação entre consumo de cafeína e BPN e não encontrou associação. O terceiro, realizado também em Campinas ${ }^{10}$, não mostrou associação entre o consumo de cafeína e BPN, prematuridade ou RCIU.

Em 1998, foram publicadas duas metanálises sobre o consumo de cafeína e desenlaces na gravidez. A primeira ${ }^{11}$ indicou uma modesta mas significante relação entre os consumos médio e alto de cafeína na gravidez e o risco de aborto espontâneo e BPN. Já a segunda ${ }^{12}$ sugeriu um provável efeito da cafeína no peso ao nascer, mas não na prematuridade. Porém, como os estudos foram muito heterogêneos entre si, essa segunda metanálise concluiu que não haveria a possibilidade de obter uma medida sumária.

Os dois estudos mais recentes foram estudos de coorte com avaliação prospectiva durante toda a gravidez sobre o consumo de cafeína e ambos não mostraram associação com a prematuridade ${ }^{13,14}$. O estudo de Bracken et al. ${ }^{14}$, entretanto, indica possibilidade de associação entre BPN e altas doses de cafeína.

\section{Fontes de cafeína e seu consumo na gravidez}

As maiores fontes de cafeína são café, chá, chocolate e refrigerantes do tipo cola ${ }^{15}$. Nesses alimentos o conteúdo de cafeína pode variar enormemente. O conteúdo de cafeína no café pode variar de 29 a $176 \mathrm{mg} /$ xícara, no chá, de 8 a $107 \mathrm{mg} /$ xícara, no chocolate de 5 a 10mg/xícara e no refrigerante do tipo cola de 32 a $65 \mathrm{mg} /$ $360 \mathrm{~mL}^{16}$.

O FDA 5 , em 1980, relatou que cerca de mil drogas prescritas e 2 mil drogas não prescritas contêm cafeína, e isso pode ser uma importante fonte para uma minoria de pessoas, particularmente para aquelas que não consomem alimentos e/ou bebidas cafeinadas ${ }^{17}$.

A cafeína pode ser encontrada em comprimidos para resfriados e alergias, e em analgésicos (15 a $64 \mathrm{mg} / \mathrm{U}$ ), moderadores de apetite (50 a 200mg/U) e estimulantes (100 a $200 \mathrm{mg} / \mathrm{U})^{18}$. Entre drogas prescritas, a dosagem 
varia de 30 a 100mg de cafeína por cápsula e entre drogas não prescritas varia de 15 a 200mg por cápsula, dependendo do tipo de produto e marca envolvida ${ }^{15}$, e 25 dessas drogas podem ser usadas na gravidez ${ }^{19}$.

A cafeína é, provavelmente, a droga mais freqüentemente ingerida no mundo, sendo consumida por pessoas em todas as idades ${ }^{20}$. 0 seu consumo é tão amplo que cerca de 95\% das mulheres grávidas ingerem alguma cafeína, seja através da alimentação ou através de medicação ${ }^{21}$.

A prevalência do consumo de cafeína durante a gravidez é relativamente alta. Em uma pesquisa nos EUA, Pastore \& Savitz ${ }^{22}$, estudando o consumo de cafeína durante a gravidez e a sua relação com a prematuridade, encontraram uma prevalência de consumo de cafeína no final da gestação de $68,1 \%$ entre os casos e $73,1 \%$ entre os controles. Fortier et al. ${ }^{23}$, no Canadá, também estudaram a mesma relação e encontraram uma prevalência de $78,4 \%$ entre a população estudada. Santos et al. ${ }^{9}$, estudando a relação entre consumo de cafeína e BPN no Sul do Brasil, encontraram uma prevalência de consumo durante a gestação de $93,0 \%$ entre os casos e $92,0 \%$ entre os controles. Camargo et al. ${ }^{24}$, analisando o consumo diário de cafeína dos produtos brasileiros (café, chá, produtos achocolatados e bebidas carbonadas) em uma amostra com 600 indivíduos de 9 a 80 anos, encontraram que $81,0 \%$ dos indivíduos consumiram regularmente refrigerante, $75,0 \%$ café, $65,0 \%$ produtos achocolatados e $37,0 \%$ chá, com uma média de consumo diário de $2,74 \mathrm{mg} / \mathrm{kg} / \mathrm{dia}$ e mediana de $1,85 \mathrm{mg} / \mathrm{kg} / \mathrm{dia}$.

O consumo diário per capita de cafeína, considerando todas as fontes, é cerca de $3-7 \mathrm{mg} /$ $\mathrm{kg} / \mathrm{dia}$, aproximadamente $200 \mathrm{mg} / \mathrm{dia}$ na população geral. Entretanto, grávidas parecem consumir menos cafeína do que outros adultos, com uma redução de $20 \%$ a $22 \%$ no consumo, que decorre principalmente da redução de café fervido ou instantâneo ${ }^{15}$. Essa redução pode estar relacionada a uma temporária perda do paladar para o café durante a gravidez ${ }^{21,25}$ e também, possivelmente, em resposta a muitos relatos publicados sobre os efeitos adversos reprodutivos.

\section{Bioquímica, fisiopatologia da cafeína e plausibilidade biológica}

A cafeína (1, 3, 7-trimetilxantina) é uma metilxantina que facilmente atravessa a barreira placentária, passando quantidades substanciais para o líquido amniótico, sangue do cordão umbilical, plasma e urina dos neonatos ${ }^{11}$. O metabolismo hepático é o mecanismo dominante de eliminação, com menos de $5 \%$ de uma dose ingerida sendo eliminada inalterada na urina. A biotransformação da cafeína é complexa, e pelo menos dezessete metabólitos urinários podem ser detectados seguindo o consumo de cafeína ${ }^{26}$. A cafeína é metabolizada à paraxantina (1,7-dimetilxantina), teofilina (1,3-dimetilxantina) e teobromina (3,7-dimetilxantina). Em humanos o principal metabólito da cafeína é a paraxantina ${ }^{27}$.

O interesse no estudo da cafeína é baseado no fato de que o clearance da cafeína é alterado durante a gravidez, sobretudo no segundo e terceiro trimestres, quando a meia-vida da cafeína é de cerca de sete e dez/onze horas, respectivamente, quando o normal é de duas horas e meia a quatro horas e meia em mulheres não grávidas $^{28}$. Esse aumento da meia-vida da cafeína nas mães coincide com o período durante o qual o desenvolvimento fetal é exponencial e então um acúmulo dessa substância pode representar um potencial risco para o feto e para a placenta, que é a responsável pela transferência de nutrientes ${ }^{29}$

Alguns mecanismos têm sido postulados para explicar tal fenômeno. A diminuição no clearance da cafeína durante a gravidez coincide com as mudanças dramáticas na concentração dos hormônios esteróides. Níveis aumentados de estrogênio e progesterona têm um efeito antagonista no metabolismo da cafeína, pois são hormônios metabolizados por enzimas hepáticas do tipo envolvidas no metabolismo das 
metilxantinas ${ }^{30,31}$. Esses hormônios levariam a um progressivo decréscimo no citocromo P-450 (enzima hepática), que é considerado, primariamente, o responsável pela desmetilação da cafeína em adultos ${ }^{32}$. Devido a isso, há um aumento da concentração de cafeína. A cafeína inibe a fosfodiesterase e essa enzima degrada a adenosina monofosfato cíclica (cAMP), elevando, dessa maneira, os níveis de CAMP, podendo interferir no crescimento e desenvolvimento das células fetais ${ }^{33}$.

A cafeína é, também, uma substância farmacologicamente ativa, com efeitos diferentes em muitos sistemas orgânicos. Os efeitos vasoconstrictores da cafeína, pelo aumento das catecolaminas (especialmente a epinefrina), podem também influenciar o desenvolvimento placentário, diminuindo o suprimento fetal de oxigênio ${ }^{34}$. Morte fetal, diminuição do peso fetal, malformação fetal e diminuição da idade gestacional podem ser conseqüências da hipóxia fetal ${ }^{35}$. Os níveis sangüíneos materno e fetal de cafeína são virtualmente o mesmo e as enzimas necessárias para o metabolismo da cafeína estão ausentes no feto e até o oitavo mês após a gravidez ${ }^{36}$.

Além disso, por causa da similaridade química entre a cafeína e os componentes de purina dos ácidos nucléicos, e, portanto, o ácido desoxirribonucléico, investigadores têm se interessado pelo potencial mutagênico da cafeína6,37. A possível incorporação de cafeína no material genético pode alterar as instruções de replicação celular ${ }^{6}$, diminuindo a fase $\mathrm{G} 2$ da mitose e, conseqüentemente, o tempo para reparo do dano no cromossomo, aumentando, dessa maneira, o percentual de células mortas ${ }^{38}$. Embora a cafeína seja mutagênica em organismos inferiores e sistemas celulares simples, seu efeito mutagênico em humanos é ainda incerto ${ }^{7}$.

\section{Limitações dos estudos sobre cafeína na associação com a prematuridade}

Dentre os principais fatores que dificultam a avaliação dos possíveis efeitos nocivos da cafeína na gravidez, podemos citar: múltiplos fatores de confusão que podem dificultar a análise do efeito da cafeína sobre a prematuridade (um dos mais importantes é o tabagismo); ampla variação no conteúdo de cafeína contido em comidas e bebidas, dificultando a obtenção de interpretações válidas de muitos estudos em humanos ${ }^{37}$; mensuração incompleta do consumo de cafeína, ou seja, não inclusão da informação sobre todas as fontes de cafeína ${ }^{11}$; possível viés de memória por parte das mães (muitos estudos foram casocontrole, nos quais a prematuridade pode afetar o relato do consumo de cafeína, particularmente se as mulheres conhecem a possibilidade de associação entre o consumo e a prematuridade)21 ; tamanho pequeno de amostra, principalmente com relação ao grupo de mulheres que consomem grandes quantidades de cafeína; e mudanças no consumo de cafeína durante a gravide $z^{27}$.

\section{CONSIDERAÇÕ ES FINAIS}

Tendo em vista as questões metodológicas levantadas nas pesquisas que avaliaram cafeína, estudos que contemplem esses aspectos, particularmente em populações com alto consumo de cafeína, e que incluam tanto prematuridade quanto BPN seriam conclusivos em relação ao efeito de alimentos-fonte de cafeína na gestação. Enquanto não houver tais estudos, parece prudente que os profissionais de saúde informem as gestantes sobre o risco de consumo elevado de alimentos-fonte de cafeína durante a gestação.

\section{REFERÊ NCIAS}

1. Terada M, Nishmura H. Mitigation of caffeine-induced teratogenicity in mice by prior chronic caffeine ingestion. Teratology. 1975; 12(1):79-87.

2. Soyka LF. Effects of methylxanthines on the fetus. Clin Perinatol. 1979; 6(1):37-51.

3. van den Berg BJ. Epidemiologic observations of prematurity: effects of tabacco, coffee and alcohol. In: Reed MD, Stanley FJ. The epidemiology of prematurity. Baltimore: Urban \& Schwarzenberg; 1997. p.157-76. 
4. Mau G, Netter P. Are coffee and alcohol consumption risk factors in pregnancy? Geburtshilfe Frauenheilkd. 1974; 34(12):1018-22.

5. Goyan JE. Food and Drug Administration. Washington (DC): FDA; 1980. p.80-36. News release; September 4; 1980.

6. Dlugosz L, Belanger K, Hellenbrand K, Holford TR, Leaderer B, Bracken MB. Maternal caffeine consumption and spontaneous abortion: a prospective cohort study. Epidemiology. 1996; 7(3):250-5.

7. Nehling A, Debry G. Potential genotoxic, mutagenic and antimutagenic effects of coffee. Review Mutat Res. 1994; 317(2):145-62.

8. Rondo PH, Rodrigues LC, Tomkins AM. Coffee consumption and intrauterine growth retardation in Brazil. Eur J Clin Nutr. 1996; 50(11):705-9.

9. Santos IS, Victora CG, Huttly S, Carvalhal JB. Caffeine intake and low birth weight: a population-based case-control study. Am J Epidemiol. 1998; 147(7):620-7.

10. Bicalho GG, Barros Filho AA. Peso ao nascer e influência do consumo de cafeína. Rev Saúde Pública. 2002; 36(2):180-7.

11. Fernandes $O$, Sabharwal M, Smiley T, Pastuszak A, Koren $\mathrm{G}$, Einarson T. Moderate to heavy caffeine consumption during pregnancy and relationship to spontaneous abortion and abnormal fetal growth: a meta-analysis. Reprod Toxicol. 1998; 12(4):435-44.

12. Santos IS, Victora CG, Huttly, Morris S. Caffeine intake and pregnancy outcomes: a meta-analytic review. Cad Saúde Pública. 1998; 14(3):523-30.

13. Clausson B, Granath F, Ekbom A, Lundgren $S$, Nordmark A, Signorello LB, et al. Effect of caffeine exposure during pregnancy on birth weight and gestational age. Am J Epidemiol. 2002; 155(5):429-36.

14. Bracken MB, Triche EW, Belanger K, Hellenbrand $\mathrm{K}$, Leaderer BP. Association of maternal caffeine consumption with decrements in fetal growth. Am J Epidemiol. 2003; 157(5):456-66.

15. Barone JJ, Roberts HR. Caffeine consumption. Food Chem Toxicol. 1996; 34(1):119-29.

16. Bunker ML, McWilliams M. Caffeine content of common beverages. J Am Diet Assoc. 1979; 74(1):28-32.

17. Bracken $M B$, Bryce-Buchanan $C$, Silten $R$, Srisuphan W. Coffee consumption during pregnancy. N Engl J Med. 1982; 306(25):1548-9.

18. Srisuphan W, Bracken MB. Caffeine consumption during pregnancy and association with late spontaneous abortion. Am J Obstet Gynecol. 1986; 154(1):14-20.

19. Srisuphan W. Caffeine consumption during pregnancy and association with miscarriage [doctoral dissertation]. Connecticut: Yale University; 1983.

20. Benowitz NL. Clinical pharmacology of caffeine. Annu Rev Med. 1990; 41:277-88.

21. Watkinson B, Fried PA. Maternal caffeine use before, during and after pregnancy and effects upon offspring. Neurobehav Toxicol Teratol. 1985; 7(1):9-17.

22. Pastore LM, Savitz DA. Case-control study of caffeinated beverages and preterm delivery. Am J Epidemiol. 1995; 141(1):61-9.

23. Fortier I, Marcoux S, Beaulac-Baillargeon L. Relation of caffeine intake during pregnancy to intrauterine growth retardation and preterm birth. Am J Epidemiol. 1993; 137(9):931-40.

24. Camargo MC, Toledo MC, Farah HG. Caffeine daily intake from dietary sources in Brazil. Food Addit Contam. 1999; 16(2):79-87.

25. Hook EB. Dietary cravings and aversions during pregnancy. Am J Clin Nutr. 1978; 31(8):1355-62.

26. Arnaud MJ. Metabolism of caffeine and other components of coffee. In: Garattini S. Caffeine, coffee and health. New York: Raven Press; 1993. p.43-95.

27. Klebanoff MA, Levine RJ, DerSimonian R, Clemens JD, Wilkins DG. Maternal serum paraxanthine, a caffeine metabolite, and the risk of spontaneous abortion. N Engl J Med. 1999; 341(22):1639-44.

28. Knutti $R$, Rothweiler $H$, Schlatter $C$. The effect of pregnancy on the pharmacokinetics of caffeine. Arch Toxicol Suppl. 1982; 5:187-92.

29. Beaulac-Baillargeon L, Desrosiers C. Caffeinecigarette interaction on fetal growth. Am J Obstet Gynecol. 1987; 157(5):1236-40.

30. Stavric B. Methylxanthines: toxicity to humans. 2. Caffeine. Food Chem Toxicol. 1988; 26(7):645-62.

31. Curatolo PW, Robertson D. The health consequences of caffeine. Ann Intern Med. 1983; 98(5 Pt 1):641-53.

32. Scott NR, Chakraborty J, Marks V. Urinary metabolites of caffeine in pregnant women. $\mathrm{Br} J$ Clin Pharmacol. 1986; 22(4):475-8.

33. Snyder SH. Adenosine as a mediator of the behavioral effects of xanthines. Berlim: SprigerVerlag; 1984.

34. Kirkinen P, Jouppila P, Koivula A, Vuori J, Puukka $M$. The effect of caffeine on placental and fetal blood flow in human pregnancy. Am J Obstet Gynecol. 1983; 147(8):939-42. 
35. Anton AH. Catecholamines during pregnancy and their effects on the fetus. Pediatr Adolesc Endocrinol. 1979; 5:110-25.

36. James JE, Paull I. Caffeine and human reproduction. Rev Environ Health. 1985; 5(2):151-67.

37. Christian MS, Brent RL. Teratogen update: evaluation of the reproductive and developmental risks of caffeine. Teratology. 2001; 64(1):51-78.

38. Gonzalez-Fernandez A, Hernandez P, Lopez-Saez JF. Effect of caffeine and adenosine on $\mathrm{G} 2$ repair: mitotic delay and chromosome damage. Mutat Res. 1985; 149(2):275-81.

39. Linn S, Schoenbaum SC, Monson RR, Rosner B, Stubblefield PG, Ryan KJ. No association between coffee consumption and adverse outcomes of pregnancy. N Engl J Med. 1982; 306(3):141-5.

40. Martin TR, Bracken MB. The association between low birth weight and caffeine consumption during pregnancy. Am J Epidemiol. 1987; 126(5):813-21.

41. Peacock JL, Bland JM, Anderson HR. Preterm delivery: effects of socioeconomic factors, psychological stress, smoking, alcohol, and caffeine. BMJ. 1995; 311(7004):531-5.

42. Wisborg K, Henriksen TB, Hedegaard M, Secher NJ. Smoking during pregnancy and preterm birth. Br J Obstet Gynaecol. 1996; 103(8):800-5.

43. Lang JM, Lieberman E, Cohen A. A comparison of risk factors for preterm labor and term small-forgestational-age birth. Epidemiology. 1996; 7(4):369-76.
44. Eskenazi B, Stapleton AL, Kharrazi M, Chee WY. Associations between maternal decaffeinated and caffeinated coffee consumption and fetal growth and gestational duration. Epidemiology. 1999; 10(3):242-9.

45. Berkowitz GS, Holford TR, Berkowitz RL. Effects of cigarette smoking, alcohol, coffee and tea consumption on preterm delivery. Early Hum Dev. 1982; 7(3):239-50.

46. Fenster L, Eskenazi B, Windham GC, Swan SH. Caffeine consumption during pregnancy and fetal growth. Am J Public Health. 1991; 81(4):458-61.

47. Olsen J, Overvad K, Frische G. Coffee consumption, birthweight, and reproductive failures. Epidemiology. 1991; 2(5):370-4.

48. Williams MA, Mittendorf R, Stubblefield PG, Lieberman E, Schoenbaum SC, Monson RR. Cigarettes, coffee, and preterm premature rupture of the membranes. Am J Epidemiol. 1992; 135(8):895-903.

49. Weathersbee PS, Olsen LK, Lodge JR. Caffeine and pregnancy. A retrospective survey. Postgrad Med. 1977; 62(3):64-9.

50. McDonald AD, Armstrong BG, Sloan M. Cigarette, alcohol, and coffee consumption and prematurity. Am J Public Health. 1992; 82(1):87-90.

Recebido para publicação em 31 de novembro de 2003 e aceito em 13 de julho de 2004. 
ANEXO 1

RESUMO DOS ESTUDOS DE COORTE PUBLICADOS SOBRE A ASSOCIAÇÃO ENTRE CAFEÍNA E PREMATURIDADE

\begin{tabular}{|c|c|c|c|c|c|}
\hline Autor(es) e ano & $n$ & Variáveis de ajuste & Fonte de cafeína & Níveis de consumo & OR (IC 95\%) \\
\hline $\begin{array}{l}\text { Van den Berg } \\
(1977)^{3}\end{array}$ & $\begin{array}{l}\text { 470/8 040 } \\
\text { (só brancos) }\end{array}$ & Nenhuma & Café & $\begin{array}{l}\leq 1 \times \text { xícara/dia } \\
2-6 \text { xícaras/dia } \\
\geq 7 \text { xícaras/dia }\end{array}$ & $\begin{array}{l}1,0 \\
1,1(1,0-1,7) \\
1,8(1,7-2,0)\end{array}$ \\
\hline $\begin{array}{l}\text { Linn et al. } \\
(1982)^{39}\end{array}$ & $860 / 10750$ & $\begin{array}{l}\text { Idade, raça, álcool, tabagismo, } \\
\text { história obstétrica, religião, es- } \\
\text { tado civil, bem-estar, escolari- } \\
\text { dade }\end{array}$ & Café & $\begin{array}{l}\text { Para } \mathrm{RPMO}^{*} \text { : } \\
<4 \text { xícaras/dia } \\
\geq 4 \text { xícaras/dia }\end{array}$ & $\begin{array}{l}1,0 \\
1,5(1,0-2,2)\end{array}$ \\
\hline $\begin{array}{l}\text { Martin \& Bracken } \\
(1987)^{40}\end{array}$ & 3881 & Nenhuma & $\begin{array}{l}\text { Café, chá, refrigerante } \\
\text { cola, medicação }\end{array}$ & $\begin{array}{l}0 \\
\geq 301 \mathrm{mg} / \mathrm{dia}\end{array}$ & $\begin{array}{l}1,0 \\
1,4(0,8-2,2)\end{array}$ \\
\hline $\begin{array}{l}\text { Fortier et al. } \\
(1993)^{23}\end{array}$ & $391 / 6342$ & $\begin{array}{l}\text { Tabagismo, renda, história obs- } \\
\text { tétrica }\end{array}$ & $\begin{array}{l}\text { Café, chá, refrigerante } \\
\text { cola, chocolate }\end{array}$ & $\begin{array}{l}0-10 \mathrm{mg} / \mathrm{dia} \\
11-150 \mathrm{mg} / \mathrm{dia} \\
151-300 \mathrm{mg} / \mathrm{dia} \\
>300 \mathrm{mg} / \mathrm{dia}\end{array}$ & $\begin{array}{l}1,0 \\
1,0(0,8-1,4) \\
1,0(0,7-1,5) \\
0,8 \quad(0,5-1,5)\end{array}$ \\
\hline $\begin{array}{l}\text { Peacock et al. } \\
(1995)^{41}\end{array}$ & $113 / 1513$ & Nenhuma & Não divulgado & $\begin{array}{l}\text { Nenhum } \\
1-1400 \mathrm{mg} / \mathrm{dia} \\
1401-2.800 \mathrm{mg} / \mathrm{dia} \\
\geq 2801 \mathrm{mg} / \mathrm{dia}\end{array}$ & $0,56^{* * * *}$ \\
\hline $\begin{array}{l}\text { Wisborg et al. } \\
(1996)^{42}\end{array}$ & $177 / 3934$ & Nenhuma & $\begin{array}{l}\text { Café, chá, chocolate e } \\
\text { refrigerante cola }\end{array}$ & $\begin{array}{l}<400 \mathrm{mg} / \mathrm{dia} \\
<400 \mathrm{mg} / \mathrm{dia}+\text { tabagis- } \\
\mathrm{mo} \\
\geq 400 \mathrm{mg} / \mathrm{dia} \\
\geq 400 \mathrm{mg} / \mathrm{dia}+\text { tabagis- } \\
\mathrm{mo}\end{array}$ & $\begin{array}{l}1,0 \\
1,0(0,6-1,5) \\
1,0 \\
2,8(1,6-4,9)\end{array}$ \\
\hline $\begin{array}{l}\text { Lang et al. } \\
(1996)^{43}\end{array}$ & 9490 & $\begin{array}{l}\text { Fatores genéticos, constitu- } \\
\text { cionais, demográficos, psicos- } \\
\text { sociais, obstétricos, nutricional, } \\
\text { pré-natal, morbidade materna, } \\
\text { exposição a tóxicos }\end{array}$ & Café ou chá & $\begin{array}{l}0 \\
1-2 \text { xícaras/dia } \\
\geq 3 \text { xícaras/dia }\end{array}$ & $\begin{array}{l}1,0 \\
1,0(0,9-1,3) \\
1,2(0,9-1,6)\end{array}$ \\
\hline $\begin{array}{l}\text { Eskenazi et } \\
\text { al.(1999)44 }\end{array}$ & 7855 & $\begin{array}{l}\text { Idade, paridade, raça, altura, es- } \\
\text { colaridade, adequação do pré- } \\
\text { natal, tabagismo no } 3^{\circ} \text { trimes- } \\
\text { tre, chá e refrigerante cola }\end{array}$ & $\begin{array}{l}\text { Café descafeinado, be- } \\
\text { bidas cafeinadas (chá, } \\
\text { café, cola) }\end{array}$ & $\begin{array}{l}\text { Não consumo } \times \text { café } \\
\text { cafeinado } \\
\text { Não consumo } \times \text { café } \\
\text { descafeinado } \\
\text { Não consumo } \times \text { café } \\
\text { cafeinado }+ \text { café } \\
\text { descafeinado }\end{array}$ & $\begin{array}{l}1,3(1,0-1,7) \\
1,1(0,7-1,7)\end{array}$ \\
\hline $\begin{array}{l}\text { Clausson et al. } \\
(2002)^{13}\end{array}$ & 873 & $\begin{array}{l}\text { Idade, IMC**, altura, país de } \\
\text { origem, filho anterior } \mathrm{C} / \mathrm{BPN}^{* * *} \text {, } \\
\text { vômito, escolaridade, trabalho, } \\
\text { náusea,fadiga, diabetes, hiper- } \\
\text { tensão }\end{array}$ & $\begin{array}{l}\text { Café, chocolate, refrige- } \\
\text { rante, medicação }\end{array}$ & $\begin{array}{l}0-99 \mathrm{mg} / \mathrm{dia} \\
100-299 \mathrm{mg} / \mathrm{dia} \\
300-499 \mathrm{mg} / \mathrm{dia} \\
\geq 500 \mathrm{mg} / \mathrm{dia}\end{array}$ & $\begin{array}{l}0,85^{* * *} \\
\text { (segundo a média } \\
\text { de } n^{\circ} \text { dias de ges- } \\
\text { tação/nível de con- } \\
\text { sumo) }\end{array}$ \\
\hline $\begin{array}{l}\text { Bracken et al. } \\
(2003)^{14}\end{array}$ & $160 / 2131$ & $\begin{array}{l}\text { Idade, paridade, número de } \\
\text { gestações, peso, situação con- } \\
\text { jugal, raça, educação, altura, } \\
\text { tabagismo no } 3^{\circ} \text { trimestre }\end{array}$ & Café, chá, soda & $\begin{array}{l}1^{\circ} \text { trimestre } \\
0 \\
1-149 \mathrm{mg} / \mathrm{dia} \\
150-299 \mathrm{mg} / \mathrm{dia} \\
\geq 300 \mathrm{mg} / \mathrm{dia} \\
3^{\circ} \text { trimestre } \\
0 \\
1-149 \mathrm{mg} / \mathrm{dia} \\
150-299 \mathrm{mg} / \mathrm{dia} \\
\geq 300 \mathrm{mg} / \mathrm{dia}\end{array}$ & $\begin{array}{l}1,0 \\
1,2(0,8-1,8) \\
1,7(0,9-3,2) \\
1,7(0,7-3,8) \\
1,0 \\
0,8(0,6-1,2) \\
1,2(0,6-2,5) \\
1,8(0,5-6,0)\end{array}$ \\
\hline
\end{tabular}

${ }^{\star} \mathrm{RPMO}=$ ruptura precoce das membranas ovulares; $* * \mathrm{IMC}=$ índice de massa corporal; $* * * B P N=$ baixo peso ao nascer; $* * * * p$ da tendência; 
ANEXO 2

RESUMO DOS ESTUDOS CASO-CONTROLE E TRANSVERSAIS PUBLICADOS SOBRE A ASSOCIAÇÃO ENTRE CAFEÍNA E PREMATURIDADE

\begin{tabular}{|c|c|c|c|c|c|}
\hline Autor (es) e ano & $\mathrm{n}$ & Variáveis de ajuste & Fonte de cafeína & Níveis de consumo & OR (IC 95\%) \\
\hline $\begin{array}{l}\text { Berkowitz et al. } \\
(1982)^{45}\end{array}$ & $\begin{array}{l}\text { Caso-controle } \\
166 / 299\end{array}$ & $\begin{array}{l}\text { Raça, peso materno, nível socioeco- } \\
\text { nômico, ganho de peso, história obs- } \\
\text { tétrica, atividade física }\end{array}$ & Café, chá & $\begin{array}{l}\text { Para o café: } \\
\text { Nenhuma } \\
1 \text { xícara } \\
2 \text { xícaras } \\
3 \text { xícaras } \\
\geq 4 \text { xícaras } \\
\text { Para o chá: } \\
\text { Nenhuma } \\
1 \text { xícara } \\
2 \text { xícaras } \\
3 \text { xícaras } \\
\geq 4 \text { xícaras }\end{array}$ & $\begin{array}{l}1,0 \\
0,6(0,4-1,1) \\
1,1(0,6-2,0) \\
1,5(0,7-3,0) \\
0,5(0,2-1,1) \\
1,0 \\
1,2(0,7-1,9) \\
1,3(0,7-2,0) \\
0,7(0,3-1,5) \\
1,5(0,8-3,1)\end{array}$ \\
\hline $\begin{array}{l}\text { Fenster et al. } \\
(1991)^{46}\end{array}$ & $\begin{array}{l}\text { Caso-controle } \\
1230\end{array}$ & $\begin{array}{l}\text { Raça, hipertensão, tabagismo, álcool, } \\
\text { educação }\end{array}$ & $\begin{array}{l}\text { Café, chá e refri- } \\
\text { gerante cola }\end{array}$ & $\begin{array}{l}\text { Total } \\
0 \\
>300 \mathrm{mg} / \mathrm{dia}\end{array}$ & $\begin{array}{l}1,3(0,6-2,7) \\
1,0 \\
1,7(0,7-4,2)\end{array}$ \\
\hline $\begin{array}{l}\text { Olsen et al. } \\
(1991)^{47}\end{array}$ & $\begin{array}{l}\text { Caso-controle } \\
370 / 11180\end{array}$ & $\begin{array}{l}\text { Tabagismo, álcool, grupo social, pari- } \\
\text { dade }\end{array}$ & Café, chá & $\begin{array}{l}\text { Para o café: } \\
\text { 0-3 xícaras/dia } \\
\text { 4-7 xícaras/dia } \\
\geq 8 \text { xícaras/dia } \\
\text { Para o chá: } \\
\text { 0-3 xícaras/dia } \\
4-7 \text { xícaras/dia } \\
\geq 8 \text { xícaras/dia }\end{array}$ & $\begin{array}{l}1,0 \\
1,1(0,9-1,4) \\
1,2(0,8-1,7) \\
1,0 \\
1,1 \quad(0,8-1,4) \\
1,6(1,0-2,7)\end{array}$ \\
\hline $\begin{array}{l}\text { Williams et al. } \\
(1992)^{48}\end{array}$ & $\begin{array}{l}\text { Caso-controle } \\
307 \mathrm{c} / \mathrm{RPMO}^{*}, 488 \\
\text { s/RPMO, } \\
2252 \text { controles }\end{array}$ & $\begin{array}{l}\text { Raça, escolaridade, idade, bem-estar, } \\
\text { situação conjugal, uso de subs. tóxi- } \\
\text { cas, paridade, tabagismo, história obs- } \\
\text { tétrica, IMC** }\end{array}$ & Café, chá & $\begin{array}{l}\text { RPMO com } \\
\geq 3 \text { xícaras/dia: cafés/ } \\
\text { RPMO com } \\
\geq 3 \text { xícaras/dia: café } \\
1^{\circ} \text { trimestre: }\end{array}$ & $\begin{array}{l}2,2(1,5-3,3) \\
1,4(1,0-1,9)\end{array}$ \\
\hline $\begin{array}{l}\text { Pastore \& Savitz } \\
(1995)^{22}\end{array}$ & $\begin{array}{l}\text { Caso-controle } \\
408 / 490\end{array}$ & $\begin{array}{l}\text { Raça, maternidade, paridade, álcool, } \\
\text { tabagismo }\end{array}$ & $\begin{array}{l}\text { Café, chá, refri- } \\
\text { gerante (cola e } \\
\text { não-cola) }\end{array}$ & $\begin{array}{l}0 \\
1-150 \mathrm{mg} / \mathrm{dia} \\
151-300 \mathrm{mg} / \mathrm{dia} \\
301-400 \mathrm{mg} / \mathrm{dia} \\
\geq 401 \mathrm{mg} / \mathrm{dia} \\
2^{\circ} \text { trimestre: } \\
0 \\
1-150 \mathrm{mg} / \mathrm{dia} \\
151-300 \mathrm{mg} / \mathrm{dia} \\
301-400 \mathrm{mg} / \mathrm{dia} \\
\geq 401 \mathrm{mg} / \mathrm{dia} \\
3^{\circ} \mathrm{trimestre:} \\
0 \\
1-150 \mathrm{mg} / \mathrm{dia} \\
151-300 \mathrm{mg} / \mathrm{dia} \\
301-400 \mathrm{mg} / \mathrm{dia} \\
\geq 401 \mathrm{mg} / \mathrm{dia}\end{array}$ & $\begin{array}{l}1,0 \\
1,3(0,9-2,0) \\
1,1(0,7-1,8) \\
0,7(0,4-1,5) \\
1,0(0,5-2,1) \\
\\
1,0 \\
1,6(1,1-2,3) \\
1,3(0,8-2,0) \\
1,0(0,5-2,0) \\
1,0(0,4-2,5) \\
\\
\\
1,0 \\
0,9(0,7-1,3) \\
0,8(0,5-1,3) \\
0,5(0,2-1,1) \\
0,6(0,3-1,4)\end{array}$ \\
\hline $\begin{array}{l}\text { Bicalho \& Barros } \\
\text { Filho }(2002)^{10}\end{array}$ & $\begin{array}{l}\text { Caso-controle } \\
354 / 354\end{array}$ & $\begin{array}{l}\text { Idade, renda, cor, escolaridade, situa- } \\
\text { ção conjugal, paridade, filhos anterio- } \\
\text { res c/BPN***, tabagismo, peso prévio } \\
\text { à gestação, trabalho, intervalo interpar- } \\
\text { tal, pré-natal, hipertensão materna }\end{array}$ & $\begin{array}{l}\text { Café, chá, } \\
\text { refrigerante }\end{array}$ & $\begin{array}{l}0 \\
<300 \mathrm{mg} / \mathrm{dia} \\
\geq 300 \mathrm{mg} / \mathrm{dia}\end{array}$ & $\begin{array}{l}1,0 \\
0,6(0,3-1,1) \\
0,3(0,2-0,7)\end{array}$ \\
\hline $\begin{array}{l}\text { Weathersbee et } \\
\text { al. }(1977)^{49}\end{array}$ & $\begin{array}{l}\text { Transversal } \\
489\end{array}$ & Nenhuma & $\begin{array}{l}\text { Café, chá, } \\
\text { refrigerante cola }\end{array}$ & $\begin{array}{l}0 \text { miligrama }(\mathrm{mg}) / \mathrm{dia} \\
\geq 300 \mathrm{mg} / \mathrm{dia}\end{array}$ & $\begin{array}{l}1,0 \\
6,4(1,7-24,1)\end{array}$ \\
\hline $\begin{array}{l}\text { McDonald et al. } \\
(1992)^{50}\end{array}$ & $\begin{array}{l}\text { Transversal } \\
2800 / 34250\end{array}$ & $\begin{array}{l}\text { Idade, peso, raça, história obstétrica, } \\
\text { tabagismo, álcool, emprego, educação }\end{array}$ & Café & $\begin{array}{l}\text { Nenhuma } \\
1-2 \text { xícaras/dia } \\
3-4 \text { xícaras/dia } \\
5-9 \text { xícaras/dia } \\
\geq 10 \text { xícaras/dia }\end{array}$ & $\begin{array}{l}1,0 \\
1,0(0,9-1,1) \\
1,1(0,9-1,2) \\
1,1(0,9-1,3) \\
1,2(0,9-1,8)\end{array}$ \\
\hline
\end{tabular}

${ }^{*} \mathrm{RPMO}=$ ruptura precoce das membranas ovulares; ${ }^{*} \mathrm{IMC}=$ índice de massa corporal; $* * * \mathrm{BPN}=$ baixo peso ao nascer. 